\section{Patient satisfaction with oral urgent treatment (OUT) in North West Tanzania}

\author{
K. Wilson, ${ }^{* 1}$ M. Bouchiba, ${ }^{2}$ G. Vithlani ${ }^{3}$ and R. Holmes ${ }^{4}$
}

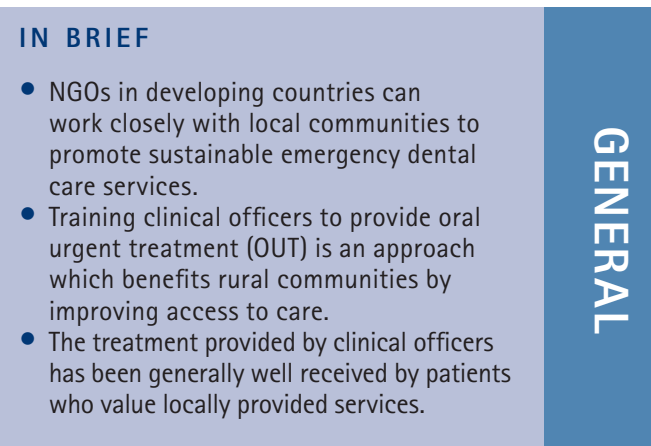

\begin{abstract}
Aim A pilot questionnaire survey was carried out to determine levels of patient satisfaction with oral urgent treatment (OUT) provided by rural clinical officers who had been trained by the charity Bridge2Aid in North West Tanzania. Method The survey was carried out over a five-day period, among adult patients who had received oral urgent treatment from a trained clinical officer. A satisfaction questionnaire was administered by a team of English-speaking dental students and Swahili-speaking college students under supervision. Results A total of 140 questionnaires were completed. The overall satisfaction with the OUT service provided by the clinical officers was high, with $93.6 \%$ being very satisfied or satisfied. However, some areas were highlighted where improvements could be made: providing a clearer explanation of treatment, improved pain control and reducing costs of treatment.
\end{abstract}

\section{INTRODUCTION}

More than 70\% of the world's population have no access to relief from dental pain. Billions of people suffer from pain in some very tough life situations. ${ }^{1}$ In developed and urbanised countries skilled professionals and available facilities enable people to access the care they require. In rural areas of East Africa access to urgent dental care is often unavailable and patients frequently have to travel to towns and cities to obtain treatment. In many cases some will simply put up with pain and infection.

There are several challenges associated with providing these patients with access to appropriate dental care but the most important consideration is to empower the local community to develop appropriate services within their healthcare structure.

\footnotetext{
Sedation Department, Newcastle School of Dental Sciences, Framlington Place, Newcastle upon Tyne, NE2 $4 A Z ;{ }^{2}$ Ferndale House Dental Practice, 250 Bromley Road Shortlands, Bromley, Kent, BR2 OBW; ${ }^{3}$ Dental Student, Guy's Hospital, St Thomas Street, London SE1 9RT; ${ }^{4}$ Centre for Oral Health Research, Newcastle School of Dental Sciences, Framlington Place, Newcastle upon Tyne, NE2 4AZ

Correspondence to: Katherine Wilson

Email:katherine.wilson@ncl.ac.uk
}

\section{Refereed Paper}

Accepted 3 June 2013

DOI: 10.1038/sj.bdj.2013.736

${ }^{\bullet}$ British Dental Journal 2013; 215: 131-134
In a recent paper $^{2}$ we described a model for providing training in oral urgent treatment (OUT) to clinical officers working at local healthcare clinics, in a region of North West Tanzania run by the organisation Bridge2Aid. As part of the evaluation of this programme it was deemed important to seek the views of the patients who had received treatment from the clinical officers, following their training. A pilot satisfaction survey was designed to record the levels of patient satisfaction with oral urgent treatment and to highlight areas for improvement in both training and service provision.

\section{METHODS}

\section{Survey design}

The work was designed as a retrospective questionnaire survey and was carried out over a five-day period in the Magu District, North West Tanzania at health centres where clinical officers had been trained.

Clinical officers had worked for at least 12 months following their training. The health centres were selected for ease of access, within a one to two hour drive from Mwanza (Bridge2Aid base). Approval for the work was gained from the Regional Medical Officer Mwanza region, Chief
Dental Officer and District Dental Officer for Magu District.

\section{Participants}

Potential participants were those who had received recent dental care by the clinical officers at the clinic, identified from the clinic register. A member of the Bridge2Aid team visited each clinic and spoke to the clinical officer and the local village leader about the survey. They were asked to locate and invite those who had had dental treatment to take part in the survey and to attend the appropriate clinic on an allocated day when the questionnaire survey would be carried out.

\section{Questionnaire}

A questionnaire was designed by one of the authors (KW) and a member of the Bridge2Aid team, to assess satisfaction with oral urgent care and it was based on work published by Kikwilu et al. ${ }^{3}$ It was translated into Kiswahili and piloted among ten people in a local community to test for appropriateness and readability.

As well as satisfaction with care, questions were included regarding the distance patients travelled to access the clinic, how patients found out about the clinic, reasons for choosing the clinic and whether they 
would return to the clinic if they needed further treatment.

\section{Administering the questionnaire}

The team administering the questionnaire was led by a UK dentist working with Bridge2Aid and included four UK dental students supported by five sixth-form students from Isamilo International School Mwanza, who acted as interpreters, and a Tanzanian Bridge2Aid senior employee.

The team travelled to the allocated clinics where patients had been requested to attend. The students worked in pairs with the Tanzanian students asking the questions and translating the answers back to the UK team members. If answers needed exploring further the UK students communicated with the assistance of the interpreters.

\section{Data management}

Data were entered into a spread sheet and analysed and presented as descriptive statistics.

\section{RESULTS}

A total of 140 questionnaires were completed and analysed. Fifty-six percent of the respondents were female and 44\% male. The age profile of the respondents is shown in Figure 1; 84\% were in the age range $18-45$ years, with $40 \%$ being between 26-35 years.

There was a range of ways in which the respondents found out about dental treatment. Information via loudspeaker (23\% respondents $n=32$ ) was the most popular method (Fig. 2). The 'other' category included announcements at church and school, and through referral by a healthcare worker

The main reason for choosing the particular health clinic visited was the close proximity to where the patient lived. 'Other' reasons are illustrated in Figure 3 and these included patients receiving a recommendation, attending the clinic for other medical reasons and through referral by a healthcare worker (some participants gave more than one reason).

Regarding levels of satisfaction with the care provided, 93.6\% of the respondents reported that they were 'very satisfied' or 'satisfied' with their dental visit. When asked if they would recommend the clinic to a friend $92 \%$ of the respondents said

Table 1 Levels of satisfaction regarding dental care

\begin{tabular}{|l|l|l|l}
\hline \multicolumn{4}{|l}{ Number of participants (\%) } \\
\hline Aspect of care & $\begin{array}{l}\text { Very satisfied/ } \\
\text { satisfied }\end{array}$ & $\begin{array}{l}\text { Neither satisfied } \\
\text { nor dissatisfied }\end{array}$ & $\begin{array}{l}\text { Dissatisfied/ } \\
\text { very dissatisfied }\end{array}$ \\
\hline Welcome by clinical officer & $134(95.8 \%)$ & $3(2.1 \%)$ & $3(2.1 \%)$ \\
\hline Cleanliness of clinic & $124(88.6 \%)$ & $10(7.1 \%)$ & $6(4.3 \%)$ \\
\hline Explanation of treatment & $111(79.3 \%)$ & $10(7.1 \%)$ & $19(13.6 \%)$ \\
\hline Pain control & $115(82.1 \%)$ & $7(5 \%)$ & $18(12.9 \%)$ \\
\hline Length of wait & $108(77.2 \%)$ & $9(6.4 \%)$ & $23(16.4 \%)$ \\
\hline Cost of treatment & $107(76.4 \%)$ & $16(11.4 \%)$ & $17(12.2 \%)$ \\
\hline Overall visit & $131(93.6 \%)$ & $3(2.1 \%)$ & $6(4.3 \%)$
\end{tabular}

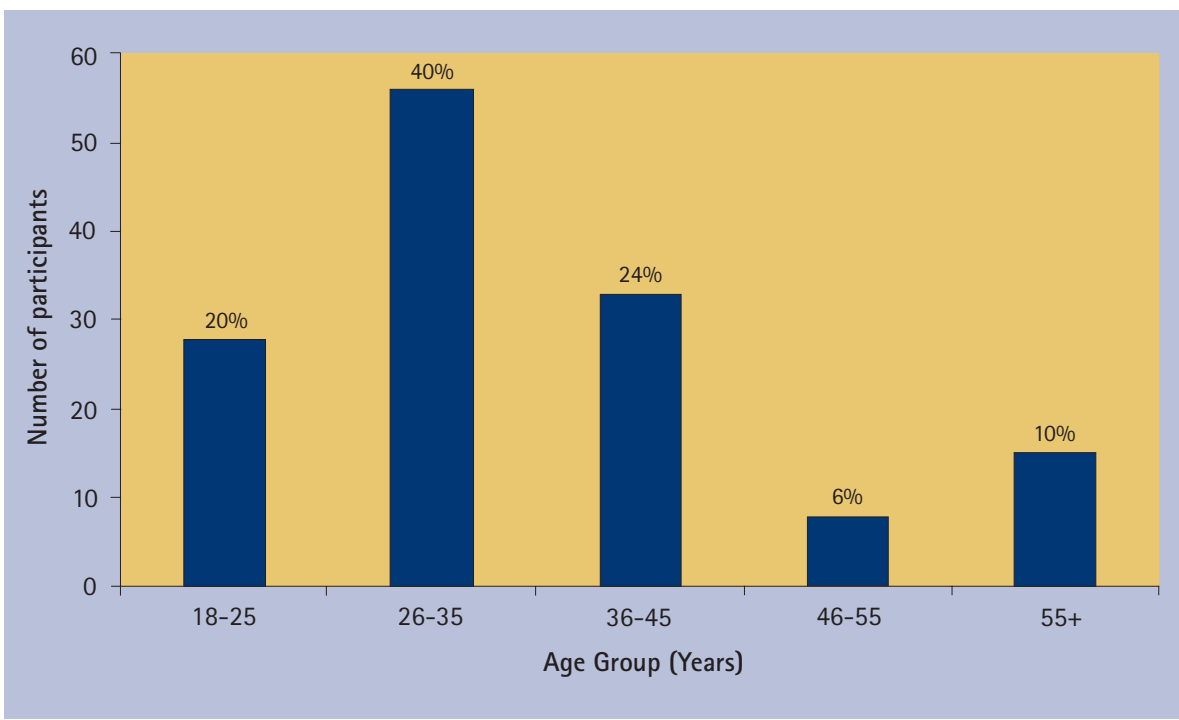

Fig. 1 Age profile of participants

yes and 93\% said they would return themselves. More specific questions were asked regarding levels of satisfaction in relation to cleanliness of the clinic, the welcome received by the clinical officer, the explanation provided about the proposed treatment, the level of pain control experienced, the length of wait and the cost of treatment. The majority of respondents, 95.8\%, were very satisfied or satisfied with the welcome received by the clinical officer; however, fewer (79.3\%) said they were 'very satisfied' or 'satisfied' with the explanation of their treatment. Pain control was generally well delivered with $82.1 \%$ of the respondents being very satisfied or satisfied with the pain control they received. Overall, 76.4\% of the respondents were very satisfied or satisfied with the cost of the treatment (Table 1).

\section{DISCUSSION}

This pilot survey has provided the first step in determining how patients perceive the care they are receiving from those trained in OUT. The results will help to inform the training providers of any issues which need to be addressed and will inform the development of a larger survey as part of the monitoring and evaluation process.

The shortfall in participant numbers was due to fewer patients attending the clinics to take part in the survey which may not be surprising as patients would have gained no direct benefit from the process. There were slightly more females $56 \%$ compared to males $44 \%$ in the sample interviewed. This may have influenced the results as some researchers have found women to be more satisfied with dental care than men..$^{3-5}$

Age may also influence how a patient perceives their dental care. Although a survey carried out by Ntabaye ${ }^{6}$ to consider satisfaction with oral care in a rural Tanzanian population found no correlation 


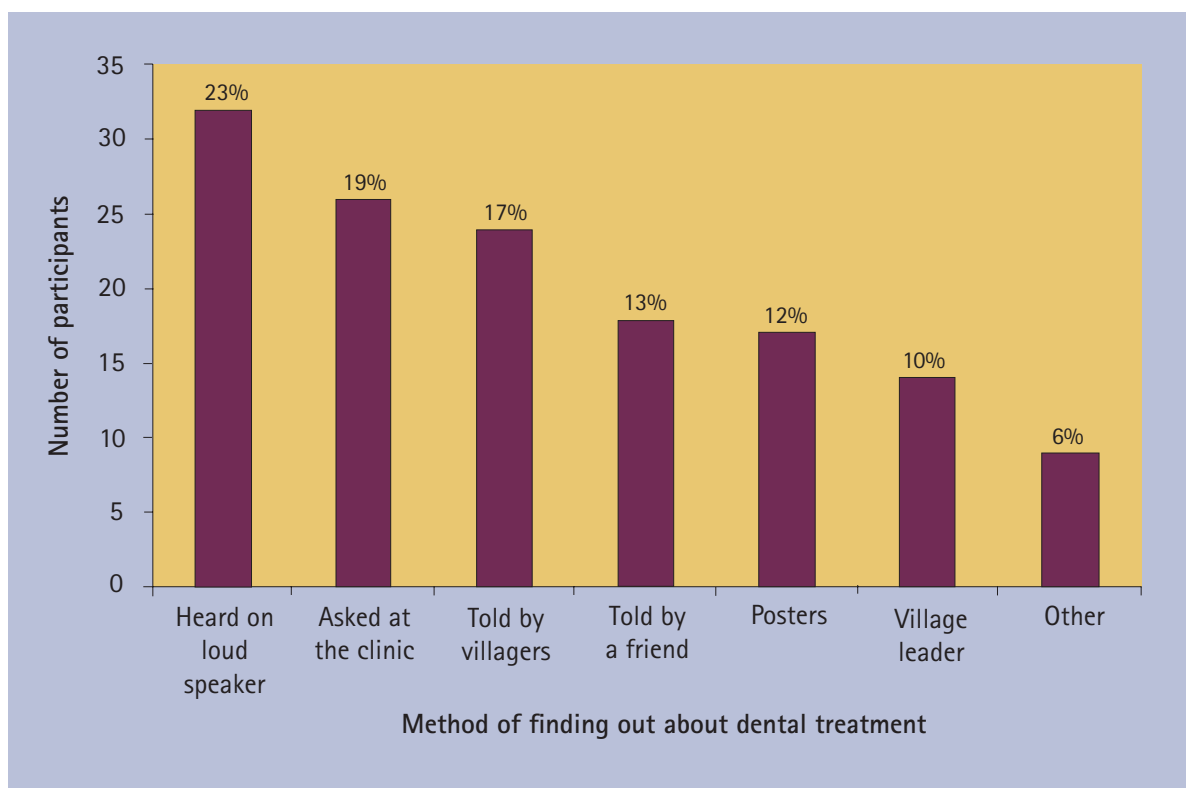

Fig. 2 Method of finding out about availability of dental treatment

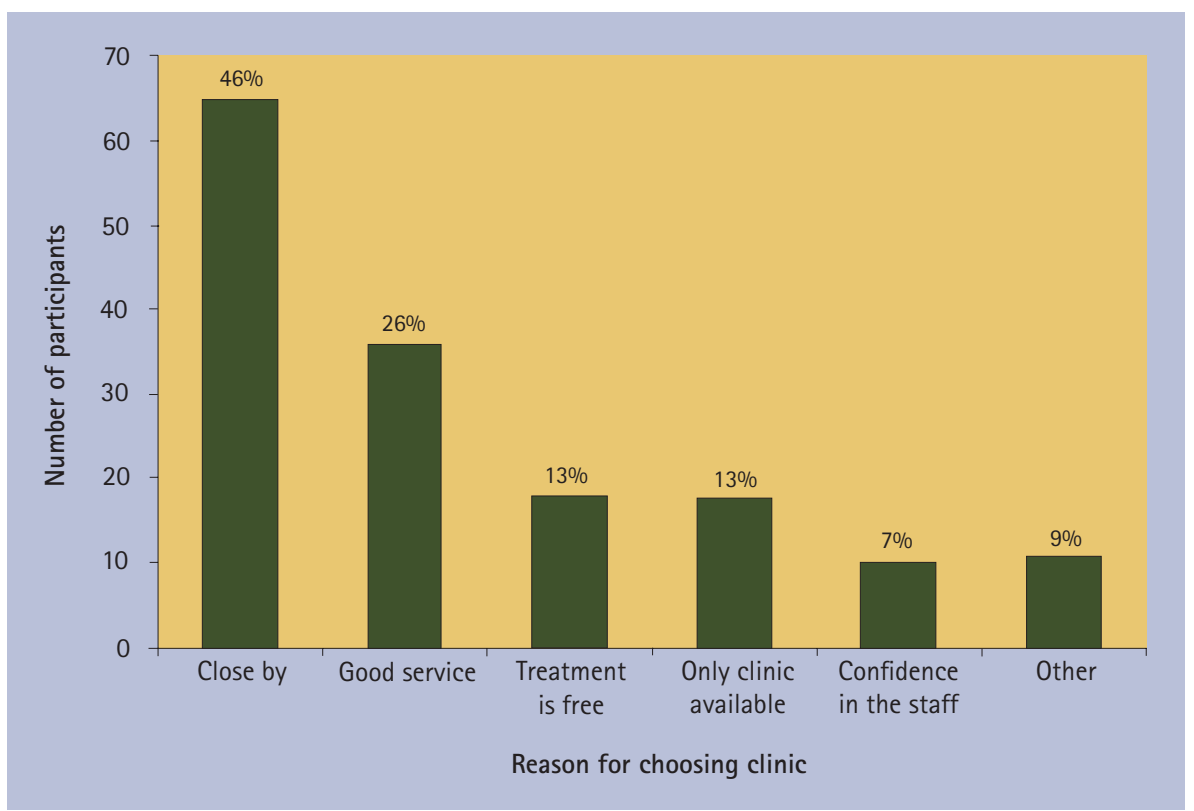

Fig. 3 Reason for choosing health clinic

with age, other studies have demonstrated that most older people tend to be more satisfied with care. ${ }^{7}$ In the present survey there were a greater number of younger people between the ages of 18 and 45 years than those aged 46 years and above, $83.6 \%$ and $16.4 \%$ respectively. It is interesting to note that fewer people in the 46-55 year age group were satisfied than expected; however, due to the small sample size it would be unwise to draw any conclusions.

A further methodological problem may have been in the delivery of the questionnaires. Respondents were asked to give their opinions directly to the interviewer and people generally like to give the answer they think is desired which could lead to an exaggerated satisfaction rate. ${ }^{6,8}$ The use of postal questionnaires may have avoided this bias however the mail service is not reliable and many rural Tanzanians have poor literacy skills and may not have been able to read and understand the questionnaire. ${ }^{3}$ An important issue for people living in rural locations is often the difficulty in accessing care. It is significant therefore, that the main reason for choosing the attended clinic was due to the close proximity to the patients' home. The main aim of the work of Bridge2Aid is to facilitate the provision of emergency dental care on a local basis and it would appear that this is being achieved.
Overall satisfaction with the dental procedure was found to be high. This may be due to the fact that all had attended the clinic due to oral pain; removing the source of the pain would have brought great relief and therefore satisfaction with care. However, when we look more closely at some of the individual aspects of the treatment visit specific concerns were raised.

Although high satisfaction rates were scored for the welcome and general management provided by the clinical officer, approximately $13.6 \%$ of patients were dissatisfied with the actual explanation of the proposed treatment. Good communication is an essential component of patient management and it is essential that the OUT training programme emphasises this to the clinical officers in training.

Not all patients were satisfied with the level of pain control they had received, $13 \%$ had found it inadequate. This compares to results reported by Kikwilu where $12.6 \%$ of patients were unhappy with the effectiveness of anaesthesia. ${ }^{3}$ Without the actual details of the dental pain history and treatment carried out it is difficult to make any definite conclusions as to the reasons for this. However, it is worthy to note that pain control is essential to ensure patient comfort and cooperation. Highlighting to training clinical officers the importance of appropriate local analgesia and the management of failed analgesia is essential.

Financial cost is an issue which often prevents people from seeking care and has been shown to have a negative effect on patient satisfaction., ${ }^{3,9}$ The current survey revealed variation in charges for care. Some clinics provided free treatment and others charged varying fees. Although the majority of patients, 76.4\%, were satisfied with the cost of their treatment approximately $12.2 \%$ were dissatisfied saying the costs were too high. Charges for urgent oral care are an issue for the government to consider and cannot be influenced directly by the service providers.

\section{SUMMARY}

The overall satisfaction with the OUT service provided by the clinical officers was generally high however some areas were highlighted where improvements could be made. 


\section{GENERAL}

It is interesting to note that the main reason behind the patients' choice of clinic was the proximity to patients' homes. This highlights the importance of ensuring services are provided locally thus improving access to care, one of the main aims of the Bridge2Aid training programme.

The main areas where patients were less satisfied were: explanation of treatment, pain control and the cost of treatment. However, the fact that 93\% would return to the clinic for dental treatment is a reflection of the general level of satisfaction and the importance of having such a service available. The areas highlighted will be addressed by the Bridge2Aid team as part of the ongoing development of the dental training programme.

The authors would like to thank the Bridge2Aid team in Tanzania without whom the survey could not have been carried out, the District Dental Officer and clinical officers who gave their time to assist in the process and to all the patients who took part and provided valuable feedback about their care. Thanks to $\mathrm{Mr}$ M. Linsley at the University of Newcastle upon Tyne for statistical advice.

1. Bridge2Aid. Online information available at www. bridge2aid.org/b2a (accessed June 2013).

2. Wilson K E, Wilson I, Holmes R D. Oral urgent treatment (OUT) - a volunteer led training programme in North West Tanzania. Br Dent J 2012: 212: 443-448.

3. Kikwilu E N, Kahabuka F K, Masalu J R, Senkoro A. Satisfaction with urgent oral care among adult Tanzanians. J Oral Sci 2009; 51: 47-54.
4. Gopalakrishna P, Mummalaneni V. Influencing satisfaction for dental services. J Health Care Mark 1993; 13: 16-22.

5. Tamaki $Y$ et al. Correlation between patient satisfaction and dental clinic credibility in regular dental check-ups in Japan. J Oral Sci 2005; 47: 97-103.

6. Ntabaye M K, Scheutz F, Poulsen S. Patient satisfaction with emergency oral health care in rural Tanzania. Community Dent Oral Epidemiol 1998; 26: 289-295.

7. Hall J A, Dornan M C. Patient socio demographic characteristics as predictors of satisfaction with medical care: a met-analysis. Soc Sci Med 2009; 30: 811-818.

8. Fitzpatrick R Hopkins A. Problems in the conceptual framework of patient satisfaction. Sociol Health IIIness 1983; 5: 297-311.

9. Stahlnacke K, Soderfeldt B, Unell L, Halling A, Axtelius B. Patient satisfaction with dental care in one Swedish cohort. Part II - What effects satisfaction. Swed Dent J 2007; 31: 137-146.

\section{Corrigendum}

General article (BDJ 2013; 214: 41-43)

'Hunterian Museum bicentenary: 1813-2013'

In the above general article, the following author was omitted in error: Milly Farrell, Curator, Hunterian Museum, Royal College of Surgeons of England, 35-43 Lincoln's Inn Fields, London, WC2A 3PE. The article was jointly written by E. Williams and M. Farrell. 